PROCEEDINGS OF THE

AMERICAN MATHEMATICAL SOCIETY

Volume 127, Number 6, Pages 1781-1788

S 0002-9939(99)04681-X

Article electronically published on February 11, 1999

\title{
AN INTERPOLATION THEOREM RELATED TO THE A.E. CONVERGENCE OF INTEGRAL OPERATORS
}

\author{
ALEXANDER KISELEV
}

(Communicated by Christopher D. Sogge)

\begin{abstract}
We show that for integral operators of general form the norm bounds in Lorentz spaces imply certain norm bounds for the maximal function. As a consequence, the a.e. convergence for the integral operators on Lorentz spaces follows from the appropriate norm estimates.
\end{abstract}

\section{INTRODUCTION}

In this paper, we study the question of a.e. convergence of integral operators satisfying certain norm estimates. The main tool in these studies is an interpolation theorem yielding the norm bounds on the maximal function of the integral operator given norm estimates on the operator itself. The result we show looks so natural and is so general that we hope it may be useful in many situations. In particular, in the Appendix we sketch the application to the spectral analysis of Schrödinger operators. It was hard to believe that the result of the kind we prove here is not known, but discussions with experts in the field convinced me that it may indeed be the case.

The main result we prove here is as follows.

Let us consider two measure spaces $(X, \mu)$ and $(Y, \nu)$ with $\mu, \nu$ being positive measures. Let $A(\lambda, x)$ be a measurable function on $X \times Y$, integrable over the sets of finite measure in $X$ for a.e. $\lambda \in Y$. Let an operator $T$ be given by

$$
(T f)(\lambda)=\int_{X} A(\lambda, x) f(x) d \mu(x) .
$$

The operator $T$ may be defined originally for all simple functions or bounded functions of finite support. Consider an expanding family of measurable regions $\Omega_{t} \subset X$, depending on the real parameter $t$, so that $\Omega_{t_{1}} \subset \Omega_{t_{2}}$ if $t_{2}>t_{1}$. Define a maximal function $M_{T}$, corresponding to the family $\left\{\Omega_{t}\right\}_{t \in R}$ and the operator $T$ by

$$
\left(M_{T} f\right)(\lambda)=\sup _{t}\left|\int_{\Omega_{t}} A(\lambda, x) f(x) d x\right| .
$$

We use the notation \|\|$_{p q}$ and \|\|$_{p q}^{*}$ for the usual norm and quasinorm in the Lorentz space $L_{p q}$ (we will recall some basic facts about these spaces below; see e.g. [1] or [7] for more information).

Received by the editors June 4, 1997 and, in revised form, September 17, 1997.

1991 Mathematics Subject Classification. Primary 42C15, 43A50; Secondary 34L40. 
Then the following theorem holds:

Theorem. Suppose that an operator T given by (1) satisfies the bounds

$$
\|T f\|_{s_{i} \infty}^{*} \leq C_{i}\|f\|_{p_{i} 1}^{*}, \quad i=1,2,
$$

where all $s_{i}, p_{i}$ are more than or equal to $1, s_{1}>s_{2}$, and $p_{1} \neq p_{2}$. Then the maximal function $M_{T}$, given by (2), satisfies

$$
\left\|\left(M_{T} f\right)\right\|_{s_{r} q} \leq C_{s}(q)\|f\|_{p_{r} q}
$$

where $s_{r}^{-1}=(1-r) s_{1}^{-1}+r s_{2}^{-1}, p_{r}^{-1}=(1-r) p_{1}^{-1}+r p_{2}^{-1}, 0<r<1$, and $q$ is any number satisfying $1 \leq q \leq \infty$.

Remark 1. The theorem above would turn into just generalized Marcinkiewicz interpolation theorem if we change $M_{T}$ to $T$ in (3).

The motivation for studying this problem comes, in particular, from the applications of a.e. convergence results for certain integral operators to the study of the spectrum of Schrödinger operators (continuous as well as discrete) [4], [5]. We briefly sketch this relationship in the Appendix, referring to the above works for details.

We note that the above theorem generalizes some old and well-known results on the a.e. convergence of the orthogonal series and Fourier integrals, in particular, the results of Paley [6] and Zygmund [8]. Of course the theorem has too general setting to touch upon the question of a.e. convergence at the corner points $(r=$ $0,1)$. These questions are very subtle even in the orthogonal series setting. In the case of trigonometric series the question about a.e. convergence of the series with coefficients from $l_{2}(Z)$ is equivalent to the celebrated Luzin's conjecture. Although in this case the a.e. convergence holds by the theorem of Carleson [2], it does not hold in general for orthonormal systems of functions (see, e.g. [3]).

\section{Proof OF THE MAIN RESUlt}

Proof. Fix a real number $\tilde{r}, 0<\tilde{r}<1$, and take $s_{3}, s_{4}$ so that $s_{1}>s_{3}>s_{\tilde{r}}>s_{4}>$ $s_{2}$. Let $p_{3}, p_{4}$ correspond to $s_{3}, s_{4}$ in a usual way. Also, fix some number $q^{\prime}$ such that $q^{\prime}>\max \left\{p_{3}, p_{4}\right\}$.

First, we prove the theorem under an additional assumption that $\mu\left(\Omega_{t}\right)$ depends continuously on $t$. The first step is to decompose the support of the function $f$ into dyadic pieces and estimate certain auxiliary maximal functions. Let $f$ be a measurable bounded function of finite support and choose $n$ so that $2^{n-1} \leq$ $\mu(\operatorname{supp}(f)) \leq 2^{n}$. Let the measurable set $E$ be the support of the function $f$ : $E=\{x|| f(x) \mid>0\}$. For every integer $m<n$, we consider a partition of the set $E$ into the dyadic pieces of size $2^{m}$ in a following way:

$$
E_{m, l}=\left(\Omega_{t_{m, l+1}} \backslash \Omega_{t_{m, l}}\right) \cap E,
$$

where $t_{m, l}$ is defined by a condition

$$
t_{m, l}=\inf \left\{t \mid \mu\left(\Omega_{t} \cap E\right)=2^{m} l\right\} .
$$

The value $t_{m, l}$ is well-defined at least for $l \leq 2^{n-m-1}$ because $\mu\left(\Omega_{t}\right)$ depends on $t$ continuously. The number of the sets $E_{m, l}$ is between $2^{n-m}$ and $2^{n-m-1}$. For notational convenience, we will assume that this number is always $2^{n-m}$ and will define the missing $E_{m, l}$ as empty sets. 
Let us define the functions $M_{m, l} f$ and $M_{m} f$ by

$$
M_{m, l} f(\lambda)=\int_{E_{m, l}} A(\lambda, x) f(x) d x \quad \text { and } \quad M_{m} f(\lambda)=\sup _{l}\left|M_{m, l} f(\lambda)\right| .
$$

Considering dyadic development of every real number $N$, it is easy to see that

$$
\left(M_{T} f\right)(\lambda) \leq \sum_{m=-\infty}^{n} M_{m} f(\lambda)
$$

Indeed, suppose that for a given value of $\lambda$, the supremum in (2) is reached when the value of the parameter $t$ is equal to $N$ (clearly if $f$ has finite support, the supremum is reached for some value of $t)$. Define the real number $a$ by $a=\mu\left(E \cap \Omega_{N}\right)$ and consider the dyadic development of $a$ :

$$
a=\sum_{j=-\infty}^{n} a_{j} 2^{j}
$$

where $a_{j}$ is equal to 0 or 1 for every $j$. Then by construction, we can find disjoint sets $E_{m, l}$, at most one for each value of $m$, so that

$$
\mu\left(\left(E \cap \Omega_{N}\right) \backslash\left(\bigcup_{m} E_{m, l}\right)\right)=0 .
$$

In fact, for each $m$ the set $E_{m, l}$ belongs to the union iff $a_{m}=1$. The corresponding value of $l$ then may be found by the formula $l=\sum_{j=m+1}^{n} a_{j} 2^{j-m}$. By the generalized Marcinkiewicz interpolation theorem (see, e.g., [1] or [7]) we have

$$
\|T f\|_{s_{r} q^{\prime}} \leq C_{r}\left(q^{\prime}\right)\|f\|_{p_{r} q^{\prime}}, 0<r<1 .
$$

Recall ([7], [1]) that the quasinorm $\|\cdot\|_{p q}^{*}$ in Lorentz spaces may be defined by (notice that when $q=p$ we get the usual $L_{p}$ norm)

$$
\|f\|_{p q}^{*}=\left(\frac{q}{p} \int_{0}^{\infty}\left|f^{*}(x)\right|^{q} x^{\frac{q}{p}-1} d x\right)^{\frac{1}{q}} .
$$

Here we denote by $f^{*}(x)$ the nonincreasing rearrangement of the function $f . f^{*}(x)$ is defined by

$$
f^{*}(x)=\inf \{y: \gamma(y) \leq x\},
$$

where $\gamma(y)$ is a distribution function of $f$ given by

$$
\gamma(y)=\mu(\{x \in M ;|f(x)|>y\}) .
$$

Let us denote by $\chi(E)$ the characteristic function of the measurable set $E$. Using the equivalence of the quasinorm $\|\cdot\|_{p q}^{*}$ and norm $\|\cdot\|_{p q}$ for $p>1$ (see, e.g., [7]), we obtain that

$$
\begin{aligned}
\left\|M_{m, l} f\right\|_{s_{i} q^{\prime}}^{q^{\prime}}= & \left\|T\left(f \chi\left(E_{m, l}\right)\right)\right\|_{s_{i} q^{\prime}}^{q^{\prime}} \leq C_{i}\left(q^{\prime}\right)^{q^{\prime}}\left(\frac{q^{\prime}}{p_{i}} \int_{0}^{\infty}\left|\left(f \chi\left(E_{m, l}\right)\right)^{*}\right|^{q^{\prime}} x^{\frac{q^{\prime}}{p_{i}}-1} d x\right) \\
& \leq C_{i}\left(q^{\prime}\right)^{q^{\prime}} 2^{m\left(\frac{q^{\prime}}{p_{i}}-1\right)} \frac{q^{\prime}}{p_{i}}\left\|f \chi\left(E_{m, l}\right)(x)\right\|_{q^{\prime}}^{q^{\prime}}, i=3,4 .
\end{aligned}
$$


We remark that since we assumed that $q^{\prime}>\max \left\{p_{3}, p_{4}\right\}$, the exponent $\frac{q^{\prime}}{p_{i}}-1$ is positive. Note that

$$
\left\|M_{m} f\right\|_{s_{i} q^{\prime}}^{q^{\prime}} \leq \sum_{l=1}^{2^{n-m}}\left\|M_{m, l} f\right\|_{s_{i} q^{\prime}}^{q^{\prime}} .
$$

Hence summing over $l$ we obtain

$$
\left\|M_{m} f\right\|_{s_{i} q^{\prime}}^{q^{\prime}} \leq C_{i}\left(q^{\prime}\right)^{q^{\prime}} \frac{q^{\prime}}{p_{i}} 2^{m\left(\frac{q^{\prime}}{p_{i}}-1\right)}\|f\|_{q^{\prime}}^{q^{\prime}}
$$

By (4), we have that

$$
\left\|M_{T} f\right\|_{s_{i} q^{\prime}} \leq C_{i}\left(q^{\prime}\right)\left(\frac{q^{\prime}}{p_{i}}\right)^{\frac{1}{q^{\prime}}}\|f\|_{q^{\prime}} \sum_{m=-\infty}^{n} 2^{m\left(\frac{1}{p_{i}}-\frac{1}{q^{\prime}}\right)}=B_{i}\left(q^{\prime}\right) 2^{n\left(\frac{1}{p_{i}}-\frac{1}{q^{\prime}}\right)}\|f\|_{q^{\prime}} .
$$

In the last inequality we denoted by $B_{i}\left(q^{\prime}\right)$ a new constant, which only depends on $s_{i}$ and $q^{\prime}$. Now we note that in a particular case when $f$ is a characteristic function of a set, $f=\chi(E),(5)$ means

$$
\left\|M_{T} \chi(E)\right\|_{s_{i} q^{\prime}} \leq B_{i}\left(q^{\prime}\right) 2^{n\left(\frac{1}{p_{i}}-\frac{1}{q^{\prime}}\right)} 2^{\frac{n}{q^{\prime}}}=B_{i}\left(q^{\prime}\right) 2^{\frac{n}{p_{i}}} \leq 2^{\frac{1}{p_{i}}} B_{i}\left(q^{\prime}\right)\|\chi(E)\|_{p_{i} 1} .
$$

It is easy to verify that the operator $M_{T} f$, defined originally on the measurable bounded functions of compact support, is a sublinear operator. We recall that it means

$$
\begin{gathered}
\left|\left(M_{T}\left(f_{1}+f_{2}\right)\right)(\lambda)\right| \leq\left|\left(M_{T} f_{1}\right)(\lambda)\right|+\left|\left(M_{T} f_{2}\right)(\lambda)\right|, \\
\left|\left(M_{T}(b f)\right)(\lambda)\right|=|b|\left|\left(M_{T} f\right)(\lambda)\right|
\end{gathered}
$$

for every scalar $b$. It is a well-known and simple to check fact that from the inequality (6) for sublinear operator it follows that $\left\|M_{T} g\right\|_{s_{i} q^{\prime}} \leq C\|g\|_{p_{i} 1}$ holds for all simple functions (see [7]) and hence by simple limiting argument for all bounded functions of finite support. Interpolating, we obtain that $\left\|M_{T} f\right\|_{s_{\tilde{r}} q} \leq C_{\tilde{r}}(q)\|f\|_{p_{\tilde{r}} q}$ for every function $f$ bounded and of compact support. It is straightforward to see that this relation is then extended to all functions $f \in L_{p_{\tilde{r}} q}$. This completes the proof under the assumption that $\mu\left(\Omega_{t}\right)$ is continuous.

Consider now the general case. The technical problem we face here is that now it is not so easy to apply the dyadic decomposition of the $\operatorname{supp}(f)=E$ into coherent pieces: the jumps of the monotone function $\mu\left(E \cap \Omega_{t}\right)$ may in general pose obstacles to that. We will handle this problem by constructing an auxiliary measure space and an auxiliary operator. We prove the estimates for the maximal function of this auxiliary operator and then show that from these estimates follows the result for the original problem.

At every value of the parameter $t$ the function $\mu\left(\Omega_{t}\right)$, as a monotone function, has limits from the left and from the right. Denote by $t_{\mp}$ the value of the jump on the left and on the right respectively: $t_{+}=\mu\left(\Omega_{t+0} \backslash \Omega_{t}\right)$ and $t_{-}=\mu\left(\Omega_{t} \backslash \Omega_{t-0}\right)$. The set of values of $t$ where any jump may occur is clearly at most countable. Let the sequence $\left\{t_{n}\right\}_{n=1}^{\infty}$ denote these points. To each $t_{n}$ corresponds the size of the jump of $\mu\left(\Omega_{t}\right)$ at this point, $y_{n}$. If for some values of $t$ we have both $t_{-}$and $t_{+}$nonzero, this value of $t$ is encountered in the above sequence twice: say, $t_{n}=t_{n+1}=t$ and $y_{n}=t_{-}, y_{n+1}=t_{+}$. Hence, for each member of the sequence $t_{n}$ only one of the two possible jumps takes place. We denote by $\Gamma_{t_{n}}$ the portion of the measure space 
corresponding to $t_{n}: \Gamma_{t_{n}}=\Omega_{t_{n}+0} \backslash \Omega_{t_{n}}$ if we have a jump on the right at $t_{n}$ and $\Gamma_{t_{n}}=\Omega_{t_{n}} \backslash \Omega_{t_{n}-0}$ otherwise.

Let us consider the following auxiliary measure space $\tilde{X}$ which we build out of $X$. At each value of $t_{n}$ we replace $\Gamma_{t_{n}} \subset X$ by

$$
B_{t_{n}}=\Gamma_{t_{n}} \times[0,1]
$$

with the structure of the product measure space. Hence

$$
\tilde{X}=\left(X \backslash\left(\bigcup_{n} \Gamma_{t_{n}}\right)\right) \cup\left(\bigcup_{m} B_{t_{m}}\right) .
$$

The measure $\tilde{\mu}$ on $\tilde{X}$ coincides with measure $\mu$ on the measurable set

$$
X_{0}=X \backslash\left(\bigcup_{n} \Gamma_{t_{n}}\right)
$$

while on $B_{t_{n}}$ the measure $\tilde{\mu}$ equals the product measure $\mu \times d x$ ( $d x$ being a Lebesgue measure on $[0,1])$. Furthermore, we let the kernel $\tilde{A}(\lambda, x)$, defined on $Y \times \tilde{X}$, be equal to $A(\lambda, x)$ for all $\lambda$ when $x \in M_{0}$ and $\tilde{A}(\lambda, x, y)=A(\lambda, x)$ for all $\lambda \in Y$ and all $y \in[0,1]$ if $x$ belongs to $\Gamma_{t_{n}}$ for some $n$. Define an integral operator $\tilde{T}$ by

$$
(\tilde{T} f)(\lambda)=\int_{\tilde{X}} \tilde{A}(\lambda, x) f(x) d x .
$$

Next, define a family $\tilde{\Omega}_{u}$ of the extending measurable sets in $\tilde{X}$. We construct $\tilde{\Omega}_{u}$ so that $\tilde{\mu}\left(\tilde{\Omega}_{u}\right)=u$. Let $t_{0}(u)=\sup _{t}\left\{t \mid \mu\left(\Omega_{t}\right) \leq u\right\}$. If $t_{0}(u) \neq t_{n}$ for any $n$, we let

$$
\tilde{\Omega}_{u}=\left(\Omega_{t_{0}(u)} \cap X_{0}\right) \cup\left(\bigcup_{t_{m}<t_{0}(u)} B_{t_{m}}\right) .
$$

In this case $\tilde{\mu}\left(\tilde{\Omega}_{u}\right)=u$, since $\mu\left(\Omega_{t}\right)$ is continuous at every $t \notin\left\{t_{n}\right\}_{n=1}^{\infty}$. Suppose now that $t_{0}(u)=t_{n}$. If at $t_{n}$ we have a jump on the left $\left(\mu\left(\Omega_{t_{n}} \backslash \Omega_{t_{n}-0}\right)>0\right)$ we let

$$
\tilde{\Omega}_{u}=\left(\Omega_{t_{n}-0} \cap X_{0}\right) \cup\left(\bigcup_{t_{m}<t_{n}} \Gamma_{t_{m}} \times[0,1]\right) \cup\left(\Gamma_{t_{n}} \times\left[0, \frac{u-\mu\left(\Omega_{t_{n}-0}\right)}{\mu\left(\Gamma_{t_{n}}\right)}\right]\right) .
$$

Otherwise, if the jump is on the right $\left(\mu\left(\Omega_{t_{n}+0} \backslash \Omega_{t_{n}}\right)>0\right)$, we define

$$
\tilde{\Omega}_{u}=\left(\Omega_{t_{n}} \cap X_{0}\right) \cup\left(\bigcup_{t_{m}<t_{n}} \Gamma_{t_{m}} \times[0,1]\right) \cup\left(\Gamma_{t_{n}} \times\left[0, \frac{u-\mu\left(\Omega_{t_{n}}\right)}{\mu\left(\Gamma_{t_{n}}\right)}\right]\right),
$$

essentially just changing $t_{n}-0$ to $t_{n}$. Defined this way, $\tilde{\Omega}_{u}$ constitutes a measurable, extending family of sets such that $\tilde{\mu}\left(\tilde{\Omega}_{u}\right)=u$ and, in particular, is continuous. Define the maximal function $\tilde{X}$, corresponding to the operator $\tilde{T}$ and the family $\tilde{\Omega}_{u}$ :

$$
\left(\tilde{M}_{\tilde{T}} f\right)(\lambda)=\sup _{u}\left|\int_{\tilde{\Omega}_{u}} \tilde{A}(\lambda, x) f(x) d \mu(x)\right|
$$


We have

Lemma. For every $r, 1>r>0$, the operator $\tilde{T}$ satisfies the norm bounds

$$
\|\tilde{T} f\|_{s_{r} p_{r}} \leq C(r)\|f\|_{p_{r}} .
$$

Proof. Indeed,

$$
\begin{aligned}
\tilde{T} f(\lambda) & =\int_{X_{0}} A(\lambda, x) f(x) d \mu(x)+\sum_{n} \int_{\Gamma_{t_{n}}} d \mu(x) \int_{0}^{1} \tilde{A}(\lambda, x, y) f(x, y) d y \\
& =\int_{X_{0}} A(\lambda, x) f(x) d \mu(x)+\sum_{n} \int_{\Gamma_{t_{n}}} d \mu(x) A(\lambda, x) \int_{0}^{1} f(x, y) d y
\end{aligned}
$$

by the definition of the kernel $\tilde{A}(\lambda, x, y)$. Because of the norm bounds on the operator $T$ and interpolation between them, we obtain

$$
\begin{aligned}
& \|\tilde{T} f\|_{L_{s_{r} p_{r}}(Y)} \leq C(r)\left(\int_{X_{0}}|f(x)|^{p_{r}} d \mu(x)+\sum_{n} \int_{\Gamma_{t_{n}}} d \mu(x)\left|\int_{0}^{1} f(x, y) d y\right|^{p_{r}}\right)^{\frac{1}{p_{r}}} \\
\leq & C(r)\left(\int_{X_{0}}|f(x)|^{p_{r}} d \mu(x)+\sum_{n} \int_{\Gamma_{t_{n}}} d \mu(x) \int_{0}^{1}|f(x, y)|^{p_{r}} d y\right)^{\frac{1}{p_{r}}} \leq C(r)\|f\|_{L_{p_{r}}(\tilde{X})} .
\end{aligned}
$$

We used Jensen inequality in the second step.

Now let us choose $r_{1}, r_{2}$ so that $s_{r_{1}}=s_{3}$ and $s_{r_{2}}=s_{4}$. By the lemma, we have the bounds

$$
\|\tilde{T} f\|_{s_{i} \infty} \leq C(i)\|f\|_{p_{i}}, i=3,4 .
$$

Since the function $\tilde{\mu}\left(\tilde{\Omega}_{u}\right)$ is continuous in $u$, we can infer from the first part of the proof that the bound

$$
\left\|\tilde{M}_{\tilde{T}}\right\|_{s_{\tilde{r}} q} \leq C_{\tilde{r}}(q)\|f\|_{p_{\tilde{r}} q}
$$

hold for every $q \in[1, \infty]$. But for every $t$, we have

$$
\int_{\Omega_{t}} A(\lambda, x) f(x) d \mu(x)=\int_{\tilde{\Omega}_{u(t)}} \tilde{A}(\lambda, y) \tilde{f}(y) d \tilde{\mu}(y) .
$$

Here $u(t)$ is such that $\mu\left(\Omega_{t}\right)=u(t)$ and $\tilde{f}$ is a function which we define as follows. If $y=x \in X_{0}$ we let $\tilde{f}(y)=f(x)$ and if $y=\left(x_{1}, y_{1}\right) \in \Gamma_{t_{n}} \times[0,1]$, we let $\tilde{f}\left(x_{1}, y_{1}\right)=f\left(x_{1}\right)$. It is easy to see that $\|\tilde{f}\|_{L_{p q}(\tilde{X})}=\|f\|_{L_{p q}(X)}$ for all $p, q$ because the distribution functions of $\tilde{f}$ and $f$ coincide. Therefore,

$$
\left(M_{T} f\right)(\lambda)=\sup _{t}\left|\int_{\Omega_{t}} A(\lambda, x) f(x) d \mu(x)\right| \leq \sup _{u}\left|\int_{\tilde{\Omega}_{u}} \tilde{A}(\lambda, y) \tilde{f}(y) d \tilde{\mu}(y)\right|=\tilde{M}_{\tilde{T}} \tilde{f}(\lambda)
$$

and hence

$$
\left\|M_{T} f\right\|_{L_{s_{\tilde{r}} q}(X)} \leq\left\|\tilde{M}_{\tilde{T}} \tilde{f}\right\|_{L_{s_{\tilde{r}} q}(\tilde{X})} \leq C_{\tilde{r}}(q)\|\tilde{f}\|_{L_{p_{\tilde{r}} q}(\tilde{X})}=C_{\tilde{r}}(q)\|f\|_{L_{p_{\tilde{r}} q}(X)} .
$$


Hence we have shown the bound (3) in the general case and since the number $\tilde{r}$ is arbitrary between 0 and 1 , the proof of the theorem is now complete.

We may now obtain the Zygmund's and Paley's theorems by specifying the measure spaces and the kernels. Let $X$ and $Y$ to be real lines equipped with Lebesgue measure and $A(\lambda, x)=\exp (i \lambda x)$. The $L_{1}-L_{\infty}$ and $L_{2}-L_{2}$ bounds are obvious and we get the Zygmund's theorem, which says that the Fourier integral converges a.e. for functions $f$ from $L_{p}, 1 \leq p<2$ and claims the corresponding estimate (3) for the maximal function.

Next let $X=Z^{1}, Y=(a, b)$ and $A(\lambda, n)=\phi_{n}(\lambda)$, where $\left\{\phi_{n}(\lambda)\right\}_{n=1}^{\infty}$ is an orthonormal uniformly bounded system in $L_{2}(a, b)$. Again the same bounds as above hold and we get the Paley's theorem which says that the series of orhtonormal functions $\sum_{n} c_{n} \phi_{n}(x)$ converges a.e. if $\left\{c_{n}\right\}_{n=1}^{\infty} \in l_{p}, 1 \leq p<2$.

The last example we would like to discuss here is that of a pseudodifferential operator in $R^{n}$ with bounded symbol $a(\lambda, x)$ :

$$
(T f)(\lambda)=\int_{R^{n}} \exp (i \lambda x) a(\lambda, x) f(x) d x .
$$

If the symbol $a(\lambda, x)$ is from the class for which an $L_{2}-L_{2}$ estimate is valid, we have that the integral defining (7) converges a.e. $\lambda$ no matter which system of extending regions $\Omega_{t}$ we take. This is the situation which appears (with $n=1$ ) in some of the applications to Schrödinger operators [4].

\section{APPENDIX}

In [4], a new approach to the investigation of the stability of the absolutely continuous spectrum of one-dimensional Schrödinger operators under slowly decreasing perturbations is developed. The absolutely continuous spectrum corresponds to the infinite motion of the quantum particle. The question of the preservation of the absolutely continuous spectrum under decaying perturbations means, roughly speaking, finding out which local, i.e. decaying, perturbations are not strong enough to destroy this infinite motion. It turns out that in many cases the problem of spectral analysis may be reduced to a problem of finding asymptotic behavior of the solutions of a certain ODE system, depending on the parameter $\lambda$, for a.e. values of this parameter ( $\lambda$ has a meaning of energy for Schrödinger equation). The latter problem may be tackled with asymptotic integration methods. The first step is an introduction of a transformation of the system, containing, in a simplest case, a function

$$
q(t, \lambda)=\int_{t}^{\infty} \theta^{2}(x, \lambda) V(x) d x .
$$

Here $\theta(x, \lambda)$ is a solution of the unperturbed Schrödinger equation, so that

$$
\left(-\frac{d^{2}}{d x^{2}}+U(x)\right) \theta(x, \lambda)=\lambda \theta(x, \lambda)
$$

and $V(x)$ is a perturbation. It turns out that if the function $q(t, \lambda)$ may be defined and decays sufficiently fast for the values of $\lambda$ from the support of the absolutely continuous spectrum of the unperturbed operator $\left(-\frac{d^{2}}{d x^{2}}+U\right)$, then this absolutely 
continuous spectrum is preserved under perturbation by $V$. For example, the simplest condition under which the a.c. spectrum is preserved is that $q(t, \lambda) V(t) \in L^{1}$ for a.e. $\lambda$ from the support of the a.c. spectrum of the unperturbed operator. The interesting case is when $V$ is not absolutely integrable; otherwise the stability is known for a long time and may be proven in a very simple way. However, although there has been a considerable attention to the subject, until recently no other general classes preserving the a.c. spectrum, given in terms of the rate of decay, were known even in the simplest situations (such as $U=0$ ).

Thus one is led to the question of studying a.e. convergence and rate of decay estimates for the integral operators like (8). The investigation of the stability of the absolutely continuous spectrum for Jacobi matrices with slowly decaying perturbations leads to similar questions.

The theorem we proved in this paper is a crucial step in the solution. It effectively reduces the problem to a simpler issue of norm estimates. Using this approach, in the paper [4] a new general class of potentials preserving the a.c. spectrum of the free $(U=0)$ and periodic ( $U$ periodic) Schrödinger operators is found. This class consists of all potentials $V$ satisfying $|V(x)| \leq C(1+x)^{-\frac{2}{3}-\epsilon}$. The applications to discrete Schrödinger operators will be considered in [5].

\section{ACKNOWLEDGMENT}

I am very grateful to Prof. S. Semmes and Prof. G. Pisier for stimulating discussions and to Prof. L. Carleson and Prof. A. Laptev for interest in this work. I also thank the referee for several useful comments and corrections. I gratefully acknowledge hospitality of IHES, where part of this work was done. Research at MSRI supported in part by NSF grant DMS 9022140.

\section{REFERENCES}

[1] J. Bergh and J. Löfström, Interpolation Spaces: An Introduction, Springer-Verlag, Berlin Heidelberg 1976. MR 58:2349

[2] L. Carleson, On convergence and growth of partial sums of Fourier series, Acta Math. 116 (1966), 135-157. MR 33:7774

[3] A. Garsia, Topics in a.e. Convergence, Markham Pub. Company, Chicago 1970. MR 41:5869

[4] A. Kiselev, Stability of the absolutely continuous spectrum of Schrödinger equation under perturbations by slowly decreasing potentials and a.e. convergence of integral operators, to appear in Duke Math. J.

[5] A. Kiselev, Stability of the absolutely continuous spectrum of Jacobi matrices under slowly decaying perturbations, in preparation.

[6] R.E.A.C. Paley, Some theorems on orthonormal functions, Studia Math. 3 (1931), 226-245.

[7] E.M. Stein and G. Weiss, Introduction to Fourier analysis on Euclidean spaces, Princeton Univ. Press, Princeton 1971. MR 46:4102

[8] A. Zygmund, A remark on Fourier transforms, Proc. Camb. Phil. Soc. 32 (1936), 321-327.

Mathematical Sciences Research Institute, 5, 1000 Centennial Drive, Berkeley, CalIFORNIA 94720

Current address: Department of Mathematics, University of Chicago, 5734 South University Avenue, Chicago, Illinois 60637-1546

E-mail address: kiselev@math.uchicago.edu 